\title{
Nitrogen excess in slowly-rotating $\beta$ Cephei stars: deep mixing or diffusion?
}

\author{
T. Morel, ${ }^{1,2}$ K. Butler, ${ }^{3}$ C. Aerts, ${ }^{1,4}$ C. Neiner, ${ }^{1,5}$ M. Briquet ${ }^{1}$ \\ ${ }^{1}$ Instituut voor Sterrenkunde, Katholieke Universiteit Leuven, Belgium \\ ${ }^{2}$ European Space Agency (ESA) postdoctoral external fellow \\ ${ }^{3}$ Universitäts-Sternwarte München, Scheinerstrasse 1, D-81679 München, Germany \\ ${ }^{4}$ Department of Astrophysics, University of Nijmegen, The Netherlands \\ ${ }^{5}$ GEPI, UMR 8111 du CNRS, Observatoire de Paris-Meudon, France
}

\begin{abstract}
We present the results of an NLTE abundance study of a small sample of $\beta$ Cephei stars, which point to the existence of a population of slowly-rotating B-type pulsators exhibiting a significant amount of nitrogen-enriched material at their surface. Although the origin of this nitrogen excess remains unclear, an overabundance preferentially occurring in stars with a detected magnetic field seems to emerge at this stage. Full details can be found in Morel et al. (2006).
\end{abstract}

\section{An abundance study of $\beta$ Cephei stars}

Precise knowledge of the fundamental parameters and metallicity of the $\beta$ Cephei stars is essential for a correct theoretical interpretation of their oscillation spectrum. This has prompted us to launch a detailed NLTE abundance analysis of nine prime targets for seismic modelling $\left(\gamma\right.$ Peg, $\delta$ Cet, $\nu$ Eri, $\beta$ CMa, $\xi^{1}$ CMa, V836 Cen, V2052 Oph, $\beta$ Cep and 12 Lac) using the line formation codes DETAIL/SURFACE and high-resolution optical spectra covering in most cases the entire oscillation cycle of the stars. A standard, iterative scheme is used to self-consistently derive the atmospheric parameters: $T_{\text {eff }}$ is determined from the silicon ionization balance, $\log g$ from fitting the collisionally-broadened wings of the Balmer lines and the microturbulence from requiring the abundances yielded by the $\mathrm{O} \mathrm{II}$ features to be independent of the line strength. All stars under study are slow rotators and curve-of-growth techniques were used to derive the abundances of $\mathrm{He}, \mathrm{C}, \mathrm{N}, \mathrm{O}, \mathrm{Mg}, \mathrm{Al}, \mathrm{Si}, \mathrm{S}$ and $\mathrm{Fe}$.

\section{On the existence of $\mathrm{N}$-rich $\beta$ Cephei stars}

The abundances of all the chemical elements (and as a result the metallicity) are indistinguishable from the values previously reported using similar techniques for early B-type dwarfs in the solar neighbourhood (e.g., Daflon \& Cunha 2004). The only notable exception is nitrogen, which appears enhanced in four targets by up to 0.6 dex. Evolutionary models including the effects of fast rotation predict that an increase of the $\mathrm{N}$ surface abundance arising from the dredge up of core-processed material should be accompanied by a strong boron depletion. Such a trend is clearly observed for the seven stars with boron data and indeed suggests that we are witnessing the results of deep mixing (see Fig. 1). However, the rotational velocities needed to account for the abundance patterns of the N-rich stars $\left(200-300 \mathrm{~km} \mathrm{~s}^{-1}\right)$ appear well in excess of the values found from seismic or line-profile variation studies $\left(<60 \mathrm{~km} \mathrm{~s}^{-1}\right)$. 


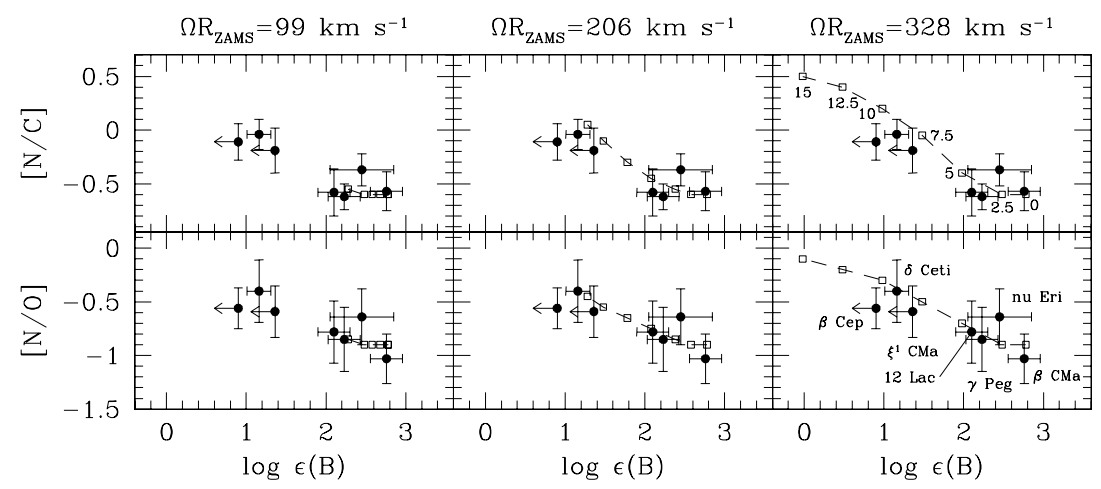

Figure 1: Ratios of the abundance of $\mathrm{C}, \mathrm{N}$ and $\mathrm{O}([\mathrm{N} / \mathrm{C}]$ and $[\mathrm{N} / \mathrm{O}])$, as a function of the NLTE boron abundances taken from the literature (Mendel et al. 2006; Proffitt \& Quigley 2001; Venn et al. 2002). These data are compared with the theoretical predictions of Heger \& Langer (2000) for a $12 \mathrm{M}_{\odot}$ star and three different values of the rotational velocity on the ZAMS: 99 (left-hand panels), 206 (middle panels) and $328 \mathrm{~km} \mathrm{~s}^{-1}$ (right-hand panels). The locus in each panel (dashed line and open squares) defines an age sequence with the time elapsed from the ZAMS increasing leftwards from $t=0$ to 15 Myrs ( $t=0$ to 12.5 Myrs for $\Omega R_{\text {ZAMS }}=99 \mathrm{~km} \mathrm{~s}^{-1}$ ) in steps of 2.5 Myrs (see upper right panel).

\section{Conclusions}

Our abundance study of several well-studied $\beta$ Cephei stars reveals in some targets an unexpected nitrogen excess systematically accompanied by a marked boron depletion. In spite of being the typical signature of rotationally-induced mixing, the existence of core-processed material brought up by this mechanism is difficult to envision for such largely unevolved, slowly-rotating objects. Alternatively, diffusion effects might be invoked (Bourge et al. 2007). An important clue to the origin of this phenomenon may lie in the fact that several $\mathrm{N}$-rich stars have a detected magnetic field of up to a few hundreds Gauss (e.g., Hubrig et al. 2006). Indeed, preliminary results also suggest similar abundance patterns in some magnetic, slowly pulsating B stars (e.g., $\zeta$ Cas; Briquet \& Morel 2007).

Acknowledgments. TM acknowledges financial support from the European Space Agency through a Postdoctoral Research Fellow grant and from the Research Council of Leuven University through grant GOA/2003/04. MB is Postdoctoral Fellow of the Fund for Scientific Research, Flanders.

\section{References}

Bourge P.-O., Théado S., Thoul A., 2007, Comm. Asteroseis., 150, 203

Briquet M., Morel T., 2007, Comm. Asteroseis., 150, 183

Daflon S., Cunha K., 2004, ApJ, 617, 1115

Heger A., Langer N., 2000, ApJ, 544, 1016

Hubrig S., Briquet M., Schöller M., et al., 2006, MNRAS, 369, L61

Mendel J. T., Venn K. A., Proffitt C. R., Brooks A. M., Lambert D. L., 2006, ApJ, 640, 1039

Morel T., Butler K., Aerts C., Neiner C., Briquet M., 2006, A\&A, 457, 651

Proffitt C. R., Quigley M. F., 2001, ApJ, 548, 429

Venn K. A., Brooks A. M., Lambert D. L., et al., 2002, ApJ, 565, 571 